\title{
ONCOLOGY PHARMACIST'S ROLE AND IMPACT ON THE MULTIDISCIPLINARY PATIENT-CENTRE PRACTICE OF ONCOLOGY CLINIC IN PUBLIC HOSPITALS IN HONG KONG
}

\author{
Chu Man Hin, Chong Chung Hong \\ United Christian Hospital, Hong Kong
}

Correspondence: cmh490@ha.org.hk

\begin{abstract}
\end{abstract}
Oncology pharmacy service was developed and integrated into the multidisciplinary team of oncology clinic in 2013 at the United Christian Hospital aiming to enhance the holistic patient-centre practice of the clinic through the optimization of the safety and efficacy of anti-cancer treatment. This review aims to describe the role and impact of oncology pharmacists (OPs) in clinical setting to optimize anti-cancer treatment for cancer patients in a multidisciplinary care approach. From selection, prescribing, procurement to monitoring and patient education, OPs significantly contribute to the safety and effective use of anti-neoplastics in any circumstances. OPs provide professional advices to oncologists in choosing the appropriate anti-cancer agents for specific cancer and designing personalized anti-cancer treatment according to patients' fitness and appropriateness for chemotherapy. Parenteral and oral chemotherapeutic agents carry heightened risk of causing significant patient harm when they are used in errors. Thus, OPs also develop standardized chemotherapy orders and ensure the final dose is appropriate in terms of both haematological and nonhaematological responses and tolerability. Moreover, OPs play an important role in procuring anti-cancer drugs and sourcing alternative drug choices that will deliver similar clinical outcomes. In addition, OPs also assure the clinical integrity of anti-cancer drugs for full anti-neoplastic activity and safe administration of these drugs by nursing staff to minimize potential occupational risk. Most importantly, OPs play a vital role in providing direct patient care functions such as drug therapy monitoring and management (e.g. ensure that patients receive sufficient pre-medications for administration of anti-cancer drugs), and medication counselling for patients and their carers to better understand their anti-cancer treatment. The positive impact of integrating OPs into the multidisciplinary patientcentre practice of oncology clinic includes (1) reduction in potentially life-threatening medication incidents and cancer drug administration errors in public hospitals; (2) collaboration with oncologists to select the most suitable cancer drug regimens for patients; (3) prevention of potential occupational risk to the healthcare professionals who handle cancer drugs; and (4) provision of optimal therapy treatment, monitoring and counselling to patients to reduce side effects and hospital readmission. The professional drug knowledge of OPs adds value to the multidisciplinary team in oncology clinics and the growth of OPs into effective direct patient care in oncology clinics should be encouraged to optimize medication-related outcomes.

\section{KEYWORDS}

oncology pharmacist, clinical pharmacist, oncology, pharmacist, anti-cancer therapy, multidisciplinary, outcome

\section{INTRODUCTION}

In Hong Kong, anti-cancer agents including both parenteral and oral agents are classified as high-alert medication in public sector, meaning they bear heightened risk of causing significant patient harm when they are used in errors. [1] Endeavour had therefore been made to avoid potential life-threatening incidents involving the anti-cancer agents. Since the role of clinical pharmacists has been well established and described in overseas setting in reducing medication errors [2-6], 
oncology clinical pharmacy service was developed to optimize the use of chemotherapy in Hong Kong.

This descriptive review paper focuses on the oncology pharmacists' (OPs) role from a viewpoint of clinical practice in an oncology centre of a public hospital in Hong Kong in various components of medication management, outlined by the Joint Commission (the accreditation body for health care facilities in the US): 1) selection; 2) procurement; 3) prescribing, dosing and transcribing; 4) storage; 5) preparing and dispensing; 6) administering; and 7) monitoring and evaluation, and 8) patient education. [7]

\section{SELECTION}

Oncology pharmacists can aid in selecting the appropriate anti-cancer agents for the specific cancer through providing up-to-date medical information. Oncologists often consult OPs for adverse effect profiles and contraindications. For instance, renal patients on renal replacement therapy such as peritoneal dialysis who are going to receive chemotherapy, OPs would discuss with oncologists to select the most appropriate regimen that are less renally excreted and recommend an appropriate dose based on the renal function. [8] More importantly, OPs would assist oncologists to design the administration schedule according to the dialysability of the prescribed regimen to optimize the treatment outcome. OPs perform drug information and literature search to provide recommendation of combination of drugs in certain diseases based on efficacy and safety concerns. OPs may also provide information on adverse effects of drugs once the oncologists suspect drug-induced problems, which aid oncologists to change and select the appropriate alternative anti-cancer agents.

During clinical screening for patients on fitness and appropriateness for chemotherapy, OPs often take into account the previous history of chemotherapy, if any, and the concurrent medication that the patient is taking. In particular, OPs calculate the cumulative anthracycline dose that the patient has taken and inform oncologists when the cumulative dose would be exceeded. [9-10] OPs collaborate with oncologists to select the appropriate medication in cancer treatment to reduce adverse effects that could happen.

Since the introduction of targeted therapy, efforts have been made to further identify the potential molecular targets for drug development. Response to targeted therapy also depends on the existence of specific gene mutation. OPs can aid in selecting the appropriate targeted therapy by screening the existence of the mutated genes and ensure the appropriate therapy is prescribed. For example, OPs would advise against the use of erlotinib or gefitinib in non-small cell lung cancer patients who acquire T790M mutation of the epidermal growth factor receptor (EGFR). [1 1] OPs also ensure that metastatic colorectal cancer patients receiving cetuximab, a recombinant human/mouse chimeric monoclonal antibody that binds specifically to the epidermal growth factor receptor (EGFR, HER 1, C-ErbB-1), are K/N-RAS wild type for the optimal treatment outcome.

\section{PROCUREMENT}

Making a drug available to the patient is certainly an essential step of pharmaceutical care. In case of drug shortages, OPs in Hong Kong play an important role in looking for alternatives of anti-cancer treatment. This may include the same drug entity with alternative drug sources or a completely different drug entity. When looking for drugs of an intrathecal treatment with different drug sources, OPs would pay particular attention to the formulation if it contains preservatives. Besides, drugs from different drug sources may require different reconstitution methods and have different volumes after reconstitution. This may affect the volume to be injected intrathecally and require careful clinical assessment. OPs would hence communicate closely with oncologists to avoid unintentional clinical incidents. If an alternative drug entity is required, OPs may need to perform dosage conversion involving anthracyclines to assess the cumulative dose administered. To enhance patient adherence and clinical outcome, OPs are also responsible for identifying drugs with suitable dosage form for medical conditions that could complicate anti-cancer treatment. Magnesium trisilicate had once been used for oral magnesium supplementation in patients experiencing hypomagnesemia in our hospital. OPs have worked to look for magnesium tablets which aid better absorption of magnesium to improve supplementation needs. Besides, folic acid solution had been used for low-dose supplementation in patients receiving pemetrexed until OPs sought out folic acid tablet with a smaller strength. This also improves patient convenience in administering the medication. 
Not only newer anti-cancer drugs are increasingly available, but also are newer chemotherapy supportive medications. Newer anti-emetics are developed to improve patient adherence through combining more than one class of medications in one pill and simplifying the regimen into single dose instead of short courses of multiple doses. OPs may work with oncologists for the availability of newer chemotherapy supportive medication in local hospitals to enhance patient convenience and hence treatment outcome.

\section{PRESCRIBING, DOSING, AND TRANSCRIBING}

Prescribing chemotherapy is a complex process involving not only the appropriate combination of drug entities but also the appropriate scheduling, dosing and route of administration. Error rates in chemotherapy have been reported at 3\%-16\%. [12-13] Standardized medication administration records (MARs) can reduce transcribing and interpretation errors and hence minimize medication errors that could be potentially life-threatening. [14] These standardized order components include diagnosis, height and weight and with the calculated body surface area (BSA), drug allergy status, dosage $(\mathrm{mg} / \mathrm{m} 2$ or $\mathrm{mg} / \mathrm{kg}$ ) with final calculated dose, start date and day of therapy, diluent solution and volume, infusion rate, route (intravenous [IV] bolus, IV infusion, subcutaneous, intramuscular or intrathecal) and the total number of scheduled doses. In our institution, standardized orders are designed by OPs which also consist of preventive antiemetic medications, hydration protocols and other essential pre-medications required. Standardized drug set in the electronic prescribing system is also designed to facilitate prescribing chemotherapy and supportive care medications by oncologists.

At the prescribing level, oncology pharmacists also screen for concurrent medical conditions that require pharmacological therapy. One particular concern in patients receiving chemotherapy is the reactivation of hepatitis B infection. In Hong Kong, the prevalence of hepatitis B carrier is around 5-10\%. [15] OPs would screen for HBsAg and anti-HBC to identify patients at high risk of hepatitis $B$ reactivation and ensure pre-emptive anti-viral treatment is given prior to initiation of chemotherapy if necessary. [16-17] To prevent herpes zoster reactivation, OPs would also recommend initiating antiviral prophylaxis such as acyclovir before starting bortezomib or daratumumab for multiple myeloma patients and throughout the treatment.

Drug-drug and drug-disease interactions influence the choice of anti-cancer drugs and the interacting drugs as well. Certain selective serotonin reuptake inhibitors (SSRIS) such as fluoxetine and paroxetine are well known CYP2D6 inhibitors which reduce the activation of tamoxifen to a more active metabolite, diminishing the anti-cancer effect. This may require modification of either the anti-cancer therapy or anti-depressants. OPs also exercise caution on prescribing injectable anti-androgens in prostate cancer patients who are concurrently taking anticoagulants as some injectable anti-androgens require intramuscular administration which is not preferred in patients on anticoagulants. Besides, OPs advise on the possibility of vaccination before and after the initiation of chemotherapy especially the administration of live attenuated vaccines.

Immunotherapy which triggers the immune system to attack tumour cells is a major advance in anti-cancer treatment in recent years. [18] Although it gives promising results in terms of disease progression and survival benefit [19-25], some patients may experience rare but potentially life-threatening immune-related adverse effects such as pneumonitis, thyroiditis and hepatitis. [26] Therefore, OPs would assure baseline monitoring of thyroid function, chest $X$-ray (CXR), liver function and other related parameters to be done before prescribing immunotherapy.

Dosing in chemotherapy requires careful calculation since some drugs such as cisplatin and pemetrexed may utilize the body surface area while some others such as trastuzumab may utilize the body weight. In calculating the dose for carboplatin, Calvert formula composing of the prescribed AUC and the creatinine clearance based on the Cockcroft-Gault Equation is used. [27] OPs play a major role in verifying the final dose given to the patients and ensure the dose prescribed does not exceed the capped dose. OPs also ensure the dose to be delivered is appropriate for renal and liver patients. If required, OPs would recommend dosing adjustment according to organ dysfunction. Besides, OPs would assess the dosing appropriateness based on patient's tolerability. Patients who experienced profound haematological toxicities may require dose adjustment in the next cycles. Significant nonhaematological toxicities such as dermatological toxicities may also warrant adjustment to improve patient's tolerance. 
Proper storage conditions with respect to temperature and lighting are essential in maintaining the chemical integrity of anti-cancer drugs for full anti-neoplastic activity. By taking reference to the manufacturer's recommendation and available drug information, OPs establish the required conditions of the reconstituted anti-cancer drugs for storage and transport. If stability data is not available for certain preparation of anti-cancer drugs, pharmacists may liaise with local laboratory to produce the relevant stability data for consideration. Besides, pharmacists are responsible for tackling look-alike and sound-alike (LASA) issues to avoid potential dispensing errors. For example, rituximab for IV infusion and for subcutaneous injection have similar packaging and require separate physical storage to avoid inadvertently incorrect picking. Tall-man lettering in the drug labels on the shelves and other auxiliary labels are also designed to differentiate between LASA drugs by highlighting the main difference in the drug names.

\section{PREPARATION AND DISPENSING}

Cytotoxic nature of anti-cancer drugs does not only cause patient harm if misused but also imposes potential occupational risk to the healthcare professionals who handle these drugs. To minimize systemic exposure to the handling personnel, reconstitution of products is best prepared in aseptic environment with the use of isolators. [28] OPs are responsible for designing standardized compounding worksheets and the effective workflow within the aseptic area that would allow timely preparation of cytotoxic drugs without compromising the quality. In particular, OPs would make sure no reconstitution of LASA drugs to be done simultaneously to avoid incidental preparation of wrong drugs. In our hospital, reconstitution of subcutaneous injection and intravenous infusion of trastuzumab are done in separate sessions, for example, one in the morning session while the other in afternoon session. OPs are also responsible for training competent pharmacy personnel to perform aseptic reconstitution. More importantly, OPs would assure the aseptic facility compliance with relevant regulatory standards and ensure safety to both pharmacists and pharmacy personnel as well as the sterility of finished products.
OPs ensure that the reconstitution of anti-cancer treatment follows manufacturer's recommendation to maintain the sterility and stability in order not to compromise the quality and effectiveness of anti-cancer drugs. Drug compatibility is one of the determinants of the physical and chemical properties of anti-cancer drugs. OPs recommend the proper diluent and material of container for preparation of anti-cancer drugs. For example, paclitaxel and docetaxel are incompatible with PVC and non-PVC bags are required to maintain the anti-neoplastic activity. OPs also ensure that light-sensitive drugs are prepared in light-protected bags. OPs also determine the expiry date on the final products by taking into account the manufacturer's recommendation and the available local laboratory testing results.

To facilitate safe administration of chemotherapy by nursing staff to the patients, OPs ensure the products are properly prepared and packaged. The final products are properly labelled and relevant auxiliary labels are affixed to remind nursing staff. Incidents involving misadministration of intrathecal vinca alkaloids had been reported worldwide. [29-31] Labels with 'For intravenous use only. Fatal if given other route.' are stuck onto the outer package of the products to further alert the use of proper route. Besides, vinca alkaloids must be prepared in at least $100 \mathrm{ml}$ infusion bags for adults to avoid intrathecal administration. In our institution, pharmacy personnel is responsible for connecting the infusion set to the infusion bags to minimize the risk of spillage in clinical area. Finished products would be packed in double-bag and properly sealed to reduce over-spillage if leakage happens. Moreover, OPs would ensure transportation of anti-cancer drugs is carried out safely. The anti-cancer drugs are transported in a designated container and must be accompanied by spillage kit to allow timely management of cytotoxic spillage by trained personnel. Cold-chain system is also strictly maintained during transportation by insulated cold packs.

\section{ADMINISTRATION}

Oncology pharmacists often receive questions on the injectable drug compatibilities with multiple infusion lines and administration sequencing from nurses. Increased availability of newer treatment has diversified anti-cancer therapy and patients may receive chemotherapy, targeted therapy and immunotherapy in the same 
regimen. OPs provide recommendation on the sequencing of drug administration to optimize the outcome of anticancer therapy. OPs provide evidence-based information to ensure safe and effective administration of cytotoxic drugs. Besides, extravasation kits and protocols are established to guide nursing staff to the appropriate management of extravasation of different drugs.

Oncology Pharmacists also design standardized medication administration form (MARs) for chemotherapy to aid the administration of the anti-cancer drugs and their supportive medications. Regimen consisting of conventional chemotherapy causes emesis to different extent. Combination of different classes of anti-emetic drugs decreases episodes of nausea and vomiting and hence enhances patients' quality of life and tolerability to cycles of anti-cancer treatment. OPs assess the emetogenicity of the anti-cancer treatment and standardize the use of anti-emetics for different regimens in the standardized MARs. This facilitates the administration of chemotherapy by reducing acute and delayed episodes of emesis. Besides, hydration protocols and premedications required are also included in the standardized MARs. For example, in the regimen consist of pemetrexed and cisplatin, pre-medication of dexamethasone and granisetron and the hydration protocols involving normal saline, mannitol, potassium and magnesium supplementation are included in the form to minimize the risk of missing necessary drugs. Infusion of monoclonal antibodies would include the relevant pre-medication such as corticosteroids, antihistamine and antipyretic to prevent anaphylactic or infusion reactions. Standardized forms have also been made specifically for intrathecal chemotherapy and only certain anti-cancer drugs which can be given by this route are permitted to be printed on these forms.

As more oral anti-cancer drugs are being developed, optimal treatment outcome becomes dependent on patient drug adherence. OPs are in a good position to educate patients on the importance of drug adherence on the treatment outcome and identify patients' concerns that would potentially dampen drug adherence. [32-35]. Equipped with professional drug knowledge, OPs are able to address any drug-drug and drug-food interactions and advise on the possible management. Drug-drug interactions between oral tyrosine kinase inhibitors (TKIs) such as gefitinib and erlotinib and gastric acid suppressants like proton pump inhibitors (PPI) and histamine-2 receptor antagonist (H2RA) are well documented and with clinical significance. Patients may require the gastric acid suppressants to treat gastrointestinal conditions such as gastric ulcer and prevent gastrointestinal complications induced by NSAIDs or steroid use. However, gastric acid suppressants may increase the gastric $\mathrm{pH}$ and lead to decreased bioavailability of TKIs. [36-38] By understanding patient's usual time of drug administration, OPs suggest the time schedule for taking TKls that would minimize the potential drug-drug interactions. OPs also examine the chronic medications which the patients are taking may be affected by the chemotherapy and its associated drugs. Patients who would proceed to transarterial chemoembolization (TACE) in hepatocellular carcinoma (HCC) may receive IV contrast during the course of treatment. OPs would remind the doctors and patients to temporarily withhold metformin to prevent contrastinduced nephropathy. Patients would be instructed to stop combination drugs which contain metformin as well.

\section{MONITORING AND EVALUATION}

Monitoring and evaluating drug therapy has long been a focusing area that clinical pharmacists can aid to optimize pharmacological therapy. Oncology pharmacists play a critical role in assessing patient's conditions before further continuing chemotherapy. OPs monitor complete blood picture, renal function and liver function in all patients receiving anti-cancer therapy and ensure parameters are within acceptable limits to proceed to the next cycle of chemotherapy. OPs may also advise the appropriate dose reduction in cases of changing renal and liver functions and altered body surface area due to significant weight changes. In patients receiving trastuzumab, OPs also monitor for multigated acquisition scan (MUGA) that measures ejection fraction and may remind physicians to order routine monitoring and temporarily withhold therapy if necessary. [39] Alanine transaminases (ALT) level is monitored in patients receiving tyrosine kinase inhibitors (TKIs) to observe for possible TKI-induced liver injury. Regular monitoring of 24-hour urine protein level before and during treatment of bevacizumab by OPs to ensure the patients are suitable to receive the treatment and prevents worsening of proteinuria after initiation of treatment.

OPs' assessment and evaluation of a patient's tolerability to previous cycles of chemotherapy can aid physicians in prescribing supportive care medicines and make slight adjustment to drug therapy. OPs may recommend prescribing pre-medication and lengthening infusion time 
in patients who experienced infusion-related reactions in previous cycles of chemotherapy. Besides, OPs may also identify episodes of nausea and vomiting in patients receiving chemotherapy with low emetogenic potential and make interventions on the use of anti-emetics. By assessing the tolerability and current performance status, OPs are always able to recommend appropriate interventions to optimize anticancer treatment.

\section{PATIENT EDUCATION}

Patient empowerment is one of the key components of successful cancer treatment. In Hong Kong, OPs deliver education through two main methods - patient education talks and one-to-one patient counselling. OPs conduct education talks in groups, covering fundamental knowledge of diseases, common anti-cancer drugs and their possible adverse effects and management methods. These talks also provide a platform for both patients who are new to chemotherapy and those who are already on chemotherapy to share their own experience and concern. At the same time, OPs would advise on some frequently-asked enquiries. By carrying out one-to-one patient counselling, OPs are able to educate both the patients and their caregivers about the appropriate use and the intention of anti-cancer treatment. Particular advice on the use of supportive care medications and any patient expectations would also be addressed in direct counselling. In our hospital, OPs would prepare patient information leaflets for oral anti-cancer drugs to facilitate patient understanding. By counteracting patients' misconceptions prior to therapy, OPs can successfully enhance patient adherence and optimize anti-cancer treatment.

Supportive care is one aspect that OPs could play an important role. This involves the use of anti-emetics to prevent chemotherapy-induced nausea and vomiting, the use of granulocyte-colony stimulating factors (GCSF) and antibiotics to prevent chemotherapy-induced neutropenic fever and certain pre-medications for chemotherapy such as pemetrexed and docetaxel. Since these medications usually have particular administration schedule, OPs would provide corresponding recommendation to facilitate patient administration. Besides, OPs would recommend patients to take steroid with famotidine to prevent gastrointestinal complications and at appropriate time to prevent sleep disturbances. Patients who will receive highly emetogenic chemotherapy are prescribed with aprepitant and require particular advice on the schedule and administration time prior to treatment. For patients receiving irinotecan, acute and chronic cholinergic adverse effects are common and may cause patient harm if not well managed, especially diarrhoea. These patients are educated by OPs to identify those acute cholinergic symptoms during administration and to manage diarrhoea by loperamide at home. Patients are also instructed to withhold capecitabine themselves if they experience grade 2 or more severe adverse reactions to minimize toxicity.

Through educating patients to avoid certain food such as grapefruit, drug-food interactions with some TKIs such as gefitinib and erlotinib can be reduced and the possibility of adverse effects can then be minimized. Besides, patients who are taking gefitinib and erlotinib are also discouraged to use over-the-counter gastric acid suppressants to avoid drug-drug interactions.

Various factors have been shown to negatively affect the drug adherence in cancer patients including therapyrelated adverse effects. [40-41] The role of OPs in educating the management of potential adverse effects is thus essential to optimal treatment outcome and better quality of life. Dermatological reactions can be present in chemotherapy, oral targeted therapy as well as immunotherapy and result in significant disturbance to the patient. These include but not limited to papulopustular rash, hand-foot skin reaction, hand-foot syndrome and paronychia. OPs can provide skin care education such as decreasing exposure to hot water, friction, avoidance of tight-fitting shoes and advice on the appropriate use of moisturizers, creams if necessary.

\section{IMPACT OF ONCOLOGY PHARMACISTS ON VARIOUS PARTIES}

Oncology pharmacy service receives great appreciation from patients, oncologists and other healthcare professionals. Patients value oncology pharmacy service as a means to medication safety and improved quality of cancer therapy. [42-43] Besides, oncology pharmacists serve as an information provider and the bridge between patients and oncologists. [42] Oncologists also value OPs as important team members and are satisfied with the improved treatment quality and safety brought by OPs. Nurses also consider OPs as important partners in improving medication safety and evaluating and managing adverse 
drug reactions. [42, 44] OPs can also reduce workload from nurses and oncologists and aid in better patient follow-up and monitoring. Cost is also saved with the input of OPs. [45] The positive ratings from different parties confirmed the beneficial service by OPs.

\section{CONCLUSION}

Oncology pharmacists are vital members of the multidisciplinary team in local hospitals of HK by offering a variety of services related to medication management to cancer patients. From selection, prescribing, procurement to monitoring and education, oncology pharmacists contribute heavily to ensure the safe and effective use of anti-cancer drugs in any circumstances. Their professional drug knowledge adds value to the multidisciplinary team and promotes rational use of anti-cancer treatment which are highly recognized by other health care professionals.

\section{References}

1. Chief Pharmacist's Office, Hospital Authority Head Office. Update of Medication Safety Guidelines issued by Medication Safety Committee. Medication Safety Bulletin. 2017; 15:1-4. Available from <https://www.ha.org.hk/haho/ho/pspa_mirp/MSB15.p df> (Accessed on 26/1 1/2018)

2. Mekonnen AB, McLachlan AJ, Brien JA. Effectiveness of pharmacist-led medication reconciliation programmes on clinical outcomes at hospital transitions: a systematic review and metaanalysis. BMJ Open. 2016 Feb 23;6(2): e010003.

3. Duarte NC, Barbosa CR, Tavares MG, et al. Clinical oncology pharmacist: Effective contribution to patient safety. J Oncol Pharm Pract. 2018:0(0):1-10.

4. Kee KW, Char CWT, Yip AYF. A review on interventions to reduce medication discrepancies or errors in primary or ambulatory care setting during care transition from hospital to primary care. J Family Med Prim Care. 2018 May-Jun;7(3):501-506.

5. Ali MAS, Khedr EMH, Ahmed FAH, Mohamed NNE. Clinical pharmacist interventions in managing drugrelated problems in hospitalized patients with neurological diseases. Int J Clin Pharm. 2018;40(5):1257-1264.

6. Drovandi A, Robertson K, Tucker M, Robinson N, Perks S, Kairuz T. A systematic review of clinical pharmacist interventions in paediatric hospital patients. Eur J Pediatr. 2018;177(8):1139-1148.

7. Joint Commission. About our standards. Available from:

$<$ http://www.jointcommission.org/standards_informati on/standards.aspx> (Accessed 26/1 1/2018)

8. Paolo Pedrazzoli 1, Nicola Silvestris, Antonio Santoro, et al. Management of patients with endstage renal disease undergoing chemotherapy: recommendations of the Associazione Italiana di Oncologia Medica (AIOM) and the Società Italiana di Nefrologia (SIN). ESMO Open. 2017;2: e000167.

9. Atiar M Rahman, Syed Wamique Yusuf, and Michael S Ewer. Anthracycline-induced cardiotoxicity and the cardiac-sparing effect of liposomal formulation. Int J Nanomedicine. 2007;2(4):567-583.

10. Menna P. and Salvatorelli E. Primary Prevention Strategies for Anthracycline Cardiotoxicity: A Brief Overview. Chemotherapy 2017; 62:159-168

11. Chunyan Ma, Shuzhen Wei and Yong Song. T790M and acquired resistance of EGFR TKI: a literature review of clinical reports. J Thorac Dis. 201 1;3(1):10-18.

12. Taylor JA, Winter L, Geyer LJ, Hawkins DS. Oral outpatient chemotherapy medications errors in children with acute lymphoblastic leukemia. Cancer. 2006; 107:1400-1406.

13. Beyzarov E. High rate of drug errors seen in pediatric oncology. Pharm Pract News. 2006; 33:10.

14. Jill N. Bryant-Bova. Improving Chemotherapy Ordering Process. Journal of Oncology Practice.2016;12(2):e248-e256.

15. Department of Health. Viral Hepatitis Preventive Service. Available:

<https://www.info.gov.hk/hepatitis/english/hep_b_set. $\mathrm{htm}>$ (Accessed 26/11/2018)

16. Jay H. Hoofnagle. Reactivation of hepatitis B. Hepatology. 2009:49(S5): S156-S165.

17. Fernando Bessone and Melisa Dirchwolf. Management of hepatitis $B$ reactivation in immunosuppressed patients: An update on current recommendations. World J Hepatol. 2016;8(8): 385394.

18. Clarence D. Moore and Irving Chen. Immunotherapy in Cancer Treatment: A Review of Checkpoint Inhibitors. US Pharm. 2018;43(2):27-31. 
19. Yang Y. Cancer immunotherapy: harnessing the immune system to battle cancer. J Clin Invest.

2015;125(9):3335-3337.

20. Meiliana A, Nurrani NM, Wijaya A. Cancer immunotherapy: a review. Indones Biomed J. 2016;8(1):1-20.

21. Farkona S, Diamandis EP, Blasutig IM. Cancer immunotherapy: the beginning of the end of cancer? BMC Med. 2016; 14:73.

22. Rosenberg SA. The emergence of modern cancer immunotherapy. Ann Surg Oncol. 2005:12(5):344-346.

23. Mellstedt H, Gaudernack G, Gerritsen WR, et al. Awareness and understanding of cancer immunotherapies in Europe. Hum Vaccin Immunother. 2014;10(7):1828-1835.

24. Reck M, Rodriguez-Abreu D, Robinson AG, et al. Pembrolizumab versus chemotherapy for PD-L 1positive-non-small cell lung cancer. N Engl J Med. 2016; 375:1823-1833.

25. Plimack E, Michaelson M, Agarwal N, et al. Expert guidance for optimizing immune checkpoint inhibitor therapy for kidney and bladder cancer. National Comprehensive Cancer Center Continuing Education. Orlando, FL: March 24, 2017.

26. American Cancer Society. What is immunotherapy? Available: <http://www.cancer.org/treatment/treatments-andside-effects/treatment-types/immunotherapy/what-isimmunotherapy.html> (Accessed 26/11/2018)

27. Van Warmerdam LJ, Rodenhuis S, ten Bokkel Huinink WW, et al. The use of the Calvert formula to determine the optimal carboplatin dosage. J Cancer Res Clin Oncol. 1995;121 (8):478-86.

28. A.C. Easty, N. Coakley, R. Cheng, et al. Safe handling of cytotoxics: guideline recommendations. Curr Oncol. 2015;22(1):e27-e37.

29. Qweider M, Gilsbach JM, Rohde V. Inadvertent intrathecal vincristine administration: a neurosurgical emergency. Case report. J Neurosurg Spine. 2007;6(3):280-3.

30. Chotsampancharoen T, Sripornsawan P, Wongchanchailert M. Two Fatal Cases of Accidental Intrathecal Vincristine Administration: Learning from Death Events. Chemotherapy. 2016;61 (2):108-10. 31. Praveena Basetty, Charlie G. Buffie, Jennifer Lagman, Cara C. Tigue, Neal Dandade and Charles L.
Bennett. Inadvertent Intrathecal Administration of Vincristine. Blood. 2006; 108:3327.

32. Noens $L$, van Lierde MA, De Bock $R$, et al. Prevalence, determinants, and outcomes of nonadherence to imatinib therapy in patients with chronic myeloid leukemia: the ADAGIO study. Blood. 2009;1 13(22):5401-11.

33. Baver S and Romvari E. Treatment of chronic myeloid leukemia following imatinib resistance: a nursing guide to second-line treatment options. Clin J Oncol Nurs. 2009;13(5):523-34.

34. Darkow T, Henk HJ, Thomas SK, et al. Treatment interruptions and non-adherence with imatinib and associated healthcare costs: a retrospective analysis among managed care patients with chronic myelogenous leukaemia. Pharmacoeconomics. 2007;25(6):481-96.

35. Vermeire $\mathrm{E}$, Hearnshaw $\mathrm{H}$, Van Royen $\mathrm{P}$, et al. Patient adherence to treatment: three decades of research. A comprehensive review. J Clin Pharm Ther. $2001 ; 26(5): 331-42$.

36. Zhang L, Wu F, Lee SC, Zhao H. pH-dependent drug-drug interactions for weak base drugs: potential implications for new drug development. Clin Pharmacol Ther. 2014; 96:266-77.

37. NR Budha, A Frymoyer, GS Smelick, et al. Drug Absorption Interactions Between Oral Targeted Anticancer Agents and PPIs: Is pH-Dependent Solubility the Achilles Heel of Targeted Therapy? Clinical Pharmacology \& Therapeutics. 2012;92(2):203213.

38. Rob ter Heine, James C. Fanggiday, Nienke A. G. Lankheet, et al. Erlotinib and pantoprazole: a relevant interaction or not? British Journal of Clinical Pharmacology. 2010;70(6):908-911.

39. Mariana Chavez-MacGregor, Jiangong Niu, Ning Zhang, et al. Cardiac Monitoring During Adjuvant Trastuzumab-Based Chemotherapy Among Older Patients with Breast Cancer. J Clin Oncol. 2015;33(19):2176-2183.

40. Jin J, Sklar GE, Min Sen Oh V, Chuen Li S. Factors affecting therapeutic compliance: A review from the patient's perspective. Ther Clin Risk Manag. 2008;4(1):269-86.

41. Guth U, Myrick ME, Schotzau A et al. Drug switch because of treatment-related adverse side effects in endocrine adjuvant breast cancer therapy: How often 
and how often does it work? Breast Cancer Res Treat 2011;129(3):799-807.

42. Yuka Sugama, Shinya Suzuki, Hayato Kamata, et al. Evaluation of Clinical Pharmacist Collaborating Service with Oncologist at Outpatient Booth in Cancer Chemotherapy from a Questionnaire Survey. J Basic Clin Pharma. 2017; 8:111-115.

43. Roxanne Dobish, Carole Chambers, Kevin Iwaasa, et al. Expanding the role of clinical pharmacists in community oncology practice: Results of implementation at the Jack Ady Cancer Clinic. OE. 2014;13(4):24-28.

44. Mancini R, Kaster $M, V \cup B$, et al. Implementation of a pharmacist-managed interdisciplinary oral chemotherapy program in a community cancer center. JHOP. $2011 ; 1$ (2):23-30.

45. Aimee DR, Douglas LS, Michael TM, et al. Is there a benefit to having a clinical oncology pharmacist on staff at a community oncology clinic? Journal of Oncology Pharmacy Practice. 201 1;17(4):425-32. 